\title{
Competência em informação em tempos de Covid-19: inovação e protagonismo no uso dos recursos informacionais
}

\section{Information literacy in Covid-19 times: innovation and protagonism in the use of informational resources}

\author{
Priscila Machado Borges Sena ${ }^{1}$, Gabriela Belmont de Farias ${ }^{2}$, Mayara Cabral Cosmo ${ }^{3}$ \\ ${ }^{1}$ Federação Brasileira de Associações de Bibliotecários, Cientistas de Informação e Instituições (FEBAB), São Paulo - SP, Brasil. ORCID: \\ http://orcid.org/0000-0002-5612-4315 \\ 2 Universidade Federal do Ceará (UFC), Fortaleza - CE, Brasil. ORCID: http://orcid.org/0000-0002-1825-0097 \\ ${ }^{3}$ Universidade Federal do Rio de Janeiro (UFRJ) Rio de Janeiro - RJ, Brasil. ORCID: http://orcid.org/0000-0002-6930-3021
}

Autor para correspondência/Mail to: Priscila Machado Borges Sena, priscilasena.pesquisa@gmail.com

Recebido/Submitted: 30 de agosto de 2020; Aceito/Approved: 03 de dezembro de 2020

Copyright (C) 2020 Sena, Farias \& Cosmo. Todo o conteúdo da Revista (incluindo-se instruções, política editorial e modelos) está sob uma licença Creative Commons Atribuição-NãoComercial-Compartilhalgual 3.0 Não Adaptada. Ao serem publicados por esta Revista, os artigos são de livre uso em ambientes educacionais, de pesquisa e não comerciais, com atribuição de autoria obrigatória. Mais informações em http://revistas.ufpr.br/atoz/about/submissions\#copyrightNotice.

\begin{abstract}
Resumo
Introdução: Busca-se relatar a experiência do webinar 'Contribuição da competência em informação no aprendizado remoto: uso dos recursos eletrônicos e midiáticos' ofertado pelo Instituto Plácido Castelo do Tribunal de Contas da União do estado do Ceará aos servidores, aos colaboradores da organização pública e à sociedade como um todo a partir das falas das pesquisadoras palestrantes. Método: Apresenta-se no relato uma abordagem metodológica de característica exploratória e descritiva, por contemplar as abordagens expostas no webinar estruturadas na dinâmica e complexidade do mundo contemporâneo; habilidades informacionais para estratégias de busca e de uso da informação; e tomada de decisão e resolução de problemas. Resultados: Alcançou-se o corpus de 223 participantes oriundos de 16 estados brasileiros, demonstrando a importância da temática, bem como a atuação social e investigativa de profissionais da Biblioteconomia e Ciência da Informação, no que concerne à comunicação e divulgação científica para a utilização além da teoria, como basilares em ações práticas mais orgânicas e contínuas na e para a sociedade. Conclusão: Evidencia-se, a partir da experiência descrita, a importância do caráter interdisciplinar da Ciência da Informação em relação a sua pertinência na sociedade por meio da divulgação científica das pesquisas realizadas e da transferência destas para a adaptação e inovação nas práticas profissionais e cotidianas.
\end{abstract}

Palavras-chave: Competência em Informação; COVID-19; Recursos informacionais; Inovação; Tecnologia; Mídias sociais

\begin{abstract}
Introduction: Introduction: We seek to report the experience of the webinar 'Contribution of competence in information in remote learning: use of electronic and media resources', offered by the Plácido Castelo Institute of the Federal Court of Accounts' State of Ceará to servers, employees of the public organization and the society, based on the speeches of the speaker researchers. Method: An exploratory and descriptive methodological approach is presented in the report, as it contemplates the approaches exposed in the webinar, structured on dynamics and complexity of the contemporary world; informational skills for search and information using strategies and decision making and problem-solving. Results: The corpus of 223 participants from 16 Brazilian states was reached, demonstrating the importance of the theme, as well as the social and investigative performance of professionals from the Library and Information Science, concerning communication and scientific dissemination for use beyond theory, as the cornerstones of more organic and continuous practical actions in and for society. Conclusions: It is evident from the experience described the importance of the interdisciplinary character of Information Science, concerning its relevance in society through the scientific dissemination of the researches carried out, and the transfer of these to adaptation and innovation in professional and daily practices.
\end{abstract}

Keywords: Information Literacy; COVID-19; Informational Resources; Innovation; Technology; Social Media

\section{INTRODUÇÃO}

O ano de 2020, que iniciou como tantos outros, expôs desafios para os quais, talvez a sociedade jamais tenha se preparado. De um momento para o outro, o mundo se viu envolvido pela pandemia do novo Coronavírus (Covid-19). As organizações públicas e privadas tiveram que se reinventar para diminuir ao máximo os efeitos devastadores instaurados pelo vírus na sociedade.

Diante desse panorama, a Escola de Contas do Tribunal de Contas do Ceará, o Instituto Plácido Castelo (IPC), organizou um evento remoto ao vivo (webinar), com o tema 'Contribuição da competência em informação no aprendizado remoto: uso dos recursos eletrônicos e midiáticos' ${ }^{1}$. A iniciativa integrou um conjunto de ações com vistas a "manter a sociedade atenta sobre os principais assuntos a partir da perspectiva atual" (Tribunal de Contas do Estado do Ceará, 2020a).

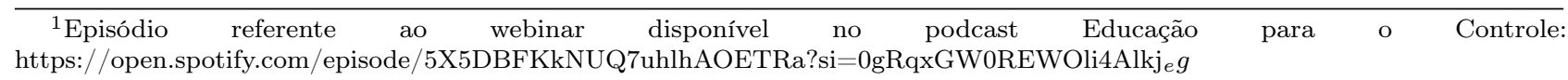


Posto isso, o webinar contou com a abordagem de três pesquisadoras bibliotecárias sobre temáticas interdisciplinares, no sentido de propiciar o compartilhamento de informação pertinente com a proposta das ações realizadas pela instituição, além de sensibilizar os seus servidores, colaboradores e interessados em atuar neste novo cenário.

Deste modo, busca-se relatar a experiência das falas das pesquisadoras na participação do webinar, ofertado a servidores, colaboradores da organização pública e à sociedade como um todo.

\section{PROCEDIMENTOS METODOLÓGICOS}

Este relato pode ser caracterizado como exploratório e descritivo, por apresentar a composição das abordagens expostas no webinar realizado no dia 05 de maio de 2020, com o objetivo incentivar os participantes a desenvolverem habilidades informacionais a partir de uma postura crítica e reflexiva sobre a realidade de acordo com: dinâmica e complexidade do mundo contemporâneo; habilidades informacionais para estratégias de busca e de uso da informação; e tomada de decisão e resolução de problemas.

Sobre as pesquisadoras convidadas pelo IPC, apresentam formação em Biblioteconomia e Pós-Graduação em Ciência da Informação com atuação no desenvolvimento de pesquisas nas referidas áreas.

A plataforma utilizada para a transmissão foi a $\mathrm{Webex}^{2}$, com a inscrição antecipada dos participantes. O evento teve duração aproximada de duas horas, incluindo a exposição do conteúdo temático pelas pesquisadoras, bem como o tempo para discussão e resposta às perguntas feitas pelos participantes.

Alcançou-se o corpus de participação de 223 pessoas oriundas de aproximadamente 16 estados brasileiros (Tribunal de Contas do Estado do Ceará, 2020b), o que ressaltou a pertinência da temática, bem como a importância de profissionais oriundos da Biblioteconomia e Ciência da Informação, no que concerne à comunicação e divulgação científica para a utilização além da teoria, como basilares em ações práticas mais orgânicas e contínuas na e para a sociedade. Nessa perspectiva, apresenta-se a síntese das abordagens que sustentaram o diálogo compartilhado no webinar.

\section{RESULTADOS}

No que concerne à inovação e tecnologia para uma Sociedade da Informação, a pesquisadora doutora Priscila Sena dialogou sobre a necessidade de compreender as configurações baseadas em inovações tecnológicas, denominadas como ecossistemas, torna-se essencial frente a pandemia da Covid-19. Principalmente quando das características de interação mútua e autonomia, presentes no conceito de ecossistema atribuído a negócios, inovação, empreendedorismo e startups. Embora distintos, esses ecossistemas possuem características e elementos em comum, como empreendedores, empresas, universidades, instituições de ciência e tecnologia, sociedade etc. (Sena, 2020).

Dessa forma, ao verificarmos o quanto a sociedade está aquém de ser considerada uma Sociedade da Informação, devido às inúmeras fragilidades postas à luz no período de pandemia, no sentido de preparo incipiente no que concerne à análise da informação, acesso e uso à informação e às tecnologias disponíveis, evidencia-se a necessidade de entender como esses clusters funcionam e interagir com eles em prol das camadas mais marginalizadas e população como um todo.

Nessa perspectiva, identificar se a cidade que residimos está pautada em um modelo de hélice tripla (governo, indústria, universidade), hélice quádrupla (governo, indústria, universidade, sociedade), hélice quíntupla (governo, indústria, universidade, sociedade, meio ambiente) pode ser um começo para buscarmos maneiras de fomentar uma sociedade mais justa, com estruturas realmente mais diversas, plurais, com igualdade de direitos e oportunidades.

Dessarte, considera-se o Tribunal de Contas do Ceará um exemplo de interação e uso positivo das inovações tecnológicas, quando da promoção de webinars e podcasts que trazem informações úteis tanto durante a pandemia como no período posterior a ela. Somado a isso, é possível trazer outras ações, como a realização de hackathons ${ }^{3}$ para ajudar bibliotecas, arquivos e museus locais a se modernizarem com relação às tecnologias que utilizam, inclusive no fomento de sistemas que promovam e auxiliem no monitoramento da quantidade de registros culturais digitalizados, o número de livros ou títulos eletrônicos de bibliotecas públicas e a porcentagem da população que é usuária desses espaços, requisito da International Organization for Standardization (2019) que estabelece indicadores internacionais para o planejamento de Cidades Inteligentes.

Ademais, é necessário estarmos abertos ao novo para além de quando estamos imersos em um período caótico, com o desenvolvimento de habilidades que nos permitam ser competentes e autônomos criticamente em relação à informação e tecnologia em todo o tipo de situação.

Do papel da Competência em Informação (CoInfo) para as atividades remotas, a pesquisadora doutora Gabriela Farias apresentou um panorama do contexto atual e os elementos que nos levam a refletir sobre as habilidades

\footnotetext{
${ }^{2}$ Mais informações em: https://www.webex.com/pt/index.html

${ }^{3}$ Mais informações em: https://pt.wikipedia.org/wiki/Hackathon
} 
em relação às tecnologias digitais de informação e comunicação (TDICs) e da atuação profissional em ambientes digitais remotos.

É necessário expor algumas razões que justificam a necessidade de desenvolver as competências em informação, pois observamos elementos que evidenciam a necessidade do indivíduo desenvolver habilidades informacionais, tais quais: o crescimento exponencial de informações disponíveis e acessíveis em qualquer formato; a informação cada vez mais heterogênea, cuja autenticidade, validade e credibilidade devem ser confirmadas; uma economia fortemente baseada em atividades de serviços, apelando a tecnologias; a necessidade de se adquirir e desenvolver habilidades transferíveis e utilizáveis ao longo da vida, aprender numa perspectiva de solução de problemas; empregadores, que querem pessoas capazes de dominar tecnologias (Bernhard, 2002). Os elementos descritos destacam a informação como pilar para a fluidez da sociedade contemporânea.

A CoInfo está relacionada aos modos ou procedimentos das práticas de informação, que tanto têm a ver com a disponibilidade de habilidades e conhecimentos instrumentais, como com as pressuposições normativas e valorativas que são pré-configuradas pelo contexto social em que o ambiente organizacional atua e do qual ele faz parte. De acordo com a Association of College and Research Libraries (2000), a CoInfo constitui-se de habilidades integradas englobando abordagem reflexiva, compreensão de como a informação é produzida e valorizada, uso da informação na criação de novos conhecimentos e participação ética em comunidades de aprendizagem.

A American Library Association (ALA) por meio da Association of College and Research Libraries (2015), publicou nove diretrizes que são universais, divididas em três categorias: competência em informação, aprendizagem independente e responsabilidade social, as quais constam no Quadro 1.

\begin{tabular}{|c|c|}
\hline CATEGORIAS & DIRETRIZES \\
\hline Competência em informação & $\begin{array}{l}\text { - Identificar a informação que deseja para } \\
\text { que tenha acesso de forma eficiente e } \\
\text { efetiva; } \\
\text { - Avalia a informação de forma crítica e } \\
\text { competente; } \\
\text { - Usa a informação de forma precisa e } \\
\text { criativa. }\end{array}$ \\
\hline $\begin{array}{l}\text { Aprendizagem independente } \\
\text { (aprender com independência) }\end{array}$ & $\begin{array}{l}\text { - Sabe buscar com persistência a } \\
\text { informação relacionada com os seus } \\
\text { interesses pessoais; } \\
\text { - Aprecia a literatura e outras formas } \\
\text { criativas de expressão da informação; } \\
\text { - Procura obter excelência na busca de } \\
\text { informação e de geração de conhecimento. }\end{array}$ \\
\hline $\begin{array}{c}\text { Responsabilidade social } \\
\text { (contribuir de modo positivo para a } \\
\text { comunidade de aprendizagem e para a } \\
\text { sociedade) }\end{array}$ & $\begin{array}{l}\text { - Reconhece a importância da informação } \\
\text { para a sociedade democrática; } \\
\text { - Coloca em prática o comportamento ético } \\
\text { em relação à informação e à tecnologia da } \\
\text { informação; } \\
\text { - Participa efetivamente de grupos para } \\
\text { buscar e gerar informação. }\end{array}$ \\
\hline
\end{tabular}

Quadro 1. Categoria da Colnfo e suas Diretrizes.

Fonte: Autoras, baseado em Association of College and Research Libraries (2015)

Competência em Informação é tão complexa e entrelaçada com a forma como se usa a informação que se devem demandar esforços para percebê-la como indivisível dos processos que constituem o nosso fazer profissional em seu contexto mais amplo (Farias \& Belluzzo, 2015).

Nesse sentido, a bibliotecária Mayara Cabral abordou sobre a necessidade do protagonismo digital para superar o distanciamento social. Pois as tecnologias surgiram a partir da necessidade de atender a uma demanda social, por consequência, isso gerou uma transformação da sociabilidade, em que diversos atores sociais se apropriam da tecnologia para manterem suas relações, pessoais, profissionais, empresariais ou institucionais, em detrimento da presença física. E tal como afirma Castells (2005), essa necessidade premente de manter tais relações nos faz viver em uma sociedade hipersocial, em que a comunicação e os processos sociais ganham uma nova dimensão com o auxílio das TDICs. Nessa perspectiva, o quadro que nos foi imposto pela pandemia global causada pela Covid-19 fez com que as tecnologias digitais assumissem um papel importante, não só quanto às relações sociais, mas também quanto ao acesso à informação, ao ganho de novas habilidades para superar a crise e para manter o funcionamento da sociedade no que for possível. 
Em relação ao uso das mídias sociais no contexto da pandemia, González-Padilla e Tortolero-Blanco (2020, p. 120) afirmam que o público leigo tem se apropriado dessas plataformas não só para o acesso à informação sobre a Covid-19, mas também como forma de manter a comunicação com os amigos e familiares, a fim de driblar o isolamento. Nesse sentido, tal como ressaltam os autores, as redes sociais são ferramentas importantes no combate aos sintomas de ansiedade e sofrimento, além de ajudar na redução do impacto de estresse psicológico causado pelo isolamento.

Assim sendo, ao analisarmos o panorama atual, algumas tendências podem ser consideradas no que diz respeito ao uso das tecnologias. O primeiro aspecto é acerca da revisão dos valores éticos e morais quanto ao uso das tecnologias e das interações digitais, o que nos traz a tônica da responsabilidade social como uma das principais demandas atuais da sociedade. Outro fator são as buscas por canais oficiais do governo, de autoridades públicas, instituições de pesquisa e de cientistas, bem como o uso de mídias sociais para a educação, conscientização e colaboração, como resposta às campanhas cada vez mais frequentes sobre desinformação e fake news.

No que se refere ao uso mais pontual das tecnologias, as tendências giram em torno da experiência do usuário, inteligência artificial e big data para tomadas de decisões estratégicas, tanto no contexto científico quanto de negócios. Por fim, relativo às mídias sociais, algumas tendências que já eram previstas para o ano de 2020 ganharam ainda mais força devido à interação frequente nas principais plataformas, como as comunidades online, a produção de conteúdo de valor para os usuários, com destaque para os formatos de vídeos e podcasts e a comunicação humanizada de marcas e instituições com o público (Terra, 2020).

Desse modo, é possível perceber que as TDICs podem ser grandes aliadas na redução dos problemas causados pela pandemia da Covid-19. O cenário de isolamento fez diversos atores sociais se apropriarem das tecnologias disponíveis para contornarem a crise e assim manterem suas relações pessoais, profissionais e institucionais. Evidenciamos que as tendências supracitadas podem auxiliar nas iniciativas de diversos profissionais, empresas e instituições, não só para minimizarem os impactos da crise, mas também para inovarem em suas respectivas áreas de atuação.

\section{CONCLUSÕES}

Com o objetivo de relatar a experiência das falas das pesquisadoras na participação do webinar ofertado a servidores, colaboradores da organização pública e à sociedade como um todo, elaborou-se este shortpaper, com vistas a integrar o dossiê temático Práticas Interdisciplinares no Contexto do Coronavírus (COVID-19), e tornar-se fonte de inspiração para novas iniciativas que envolvem a temática.

O verdadeiro protagonismo surge quando os indivíduos são capacitados e, com acesso ao conhecimento que lhe é de direito, problematizam sua realidade, identificam os desafios que interferem em seu cotidiano e as causas que o mobilizam, e traçam caminhos para superá-los, por meio de suas habilidades informacionais. Compreendemos que os ambientes digitais, ainda que de maneira informal, possibilitam formação e desenvolvimento de profissionais por meio do acesso à informação e interatividade; entretanto, o papel das instituições é de não somente absorver essa realidade como também apresentar e trabalhar de forma inovadora com as diversas possibilidades que o meio digital oferece, nutrindo colaboradores mais engajados.

Evidencia-se, a partir da experiência descrita, a importância do caráter interdisciplinar da Ciência da Informação, no que concerne a sua pertinência em sociedade por meio da divulgação científica das pesquisas realizadas, e da transferência destas para a adaptação e inovação por intermédio da reinvenção de práticas profissionais e cotidianas.

\section{AGRADECIMENTOS}

À Escola de Contas do Tribunal de Contas do Ceará, o Instituto Plácido Castelo (IPC) pela iniciativa e organização do webinar 'Contribuição da competência em informação no aprendizado remoto: uso dos recursos eletrônicos e midiáticos'. 


\section{REFERÊNCIAS}

Association of College and Research Libraries . (2000). Information literacy competency standards for higher education. Chicago, IL: ACRL. Recuperado de http://www.ala.org/ acrl/ilintro.html

Association of College and Research Libraries . (2015). Framework for information literacy for higher education. Chicago, IL: ACRL. Recuperado de http://www.ala.org/ acrl/standards/ilframework

Bernhard, P. (2002). La formación en el uso de la información: una ventaja en la enseñanza superior. situación actual. In Anales de documentación (v. 5, p. 409-435). Recuperado de https://revistas.um.es/analesdoc/article/view/2271

Castells, M. (2005). A sociedade em rede: do conhecimento à política. In $A$ sociedade em rede: do conhecimento à ação política (p. 17-30). Belém, Portugal: Imprensa Naciona; Casa da Moeda. Recuperado de http://egov.ufsc.br/portal/conteudo/sociedade-em-rede -do-conhecimento-à-acç $\sim$ ao-política

Farias, G. B., \& Belluzzo, R. C. B. (2015). Como desenvolver a competência em informação mediada por modelagem conceitual teórico-prática: por uma aprendizagem significativa e criativa na educação. Londrina: ABECIN Editora.

González-Padilla, D. A., \& Tortolero-Blanco, L. (2020). Social media influence in the covid-19 pandemic. International Brazilian Journal of Urology, 46(supl.1). Recuperado de https://www.scielo.br/scielo.php?script=sci _arttext\&pid=S1677-55382020000700120\&tlng=en

International Organization for Standardization. (2019). Iso 37120: sustainable cities and communities: indicators for smart cities. Recuperado de https://www.iso.org/standard/ 69050.html

Sena, P. M. B. (2020). Fontes de informação no ecossistema de startups de florianápolis (Tese de Doutorado, Universidade Federal de Santa, Florianápolis, SC, Brasil). Recuperado de http://tede.ufsc.br/teses/PCIN0226-T.pdf

Terra, R. (2020). 25 tendências de marketing digital e redes sociais para 2020. Recuperado de https://www .mundodomarketing.com.br/

Tribunal de Contas do Estado do Ceará. (2020a). Contribuição da competência em informação no aprendizado remoto. Recuperado de https://www.tce.ce.gov.br/comunicacao/ noticias/4107-ipc-promove-webinar-sobre-contribuicao-da -competencia-em-informacao-no-aprendizado-remoto

Tribunal de Contas do Estado do Ceará. (2020b). O uso da informação válida e confível para tomadas de decisões foi destaque em webinar do ipc. Recuperado de https://www.tce.ce.gov.br/comunicacao/noticias/ 4117-o-uso-da-informacao-valida-e-confiavel-para-tomadas -de-decisoes-foi-destaque-em-webinar-do-ipc

Sena, P. M. B., Farias, G. B. \& Cosmo, M. C. (2020). Competência em informação em tempos de Covid-19: inovação e protagonismo no uso dos recursos informacionais. AtoZ: novas práticas em informação e conhecimento, 9(2), 253 - 257. Recuperado de: http:// dx.doi.org/10.5380/atoz.v9i2.76149 